\title{
Solving the dark-matter problem through dynamic interactions
}

\author{
Werner A. Hofer \\ School of Mathematics and Statistics, Newcastle University, Newcastle upon Tyne NE1 7RU, UK \\ E-mail: werner.hofer@ncl.ac.uk \\ Received July 21, 2015; accepted September 24, 2015
}

\begin{abstract}
Owing to the renewed interest in dark matter after the upgrade of the large hadron collider and its dedication to dark-matter research, it is timely to reassess the whole problem. Considering dark matter is one way to reconcile the discrepancy between the velocity of matter in the outer regions of galaxies and the observed galactic mass. Thus far, no credible candidate for dark matter has been identified. Here, we develop a model accounting for observations by rotations and interactions between rotating objects analogous to magnetic fields and interactions with moving charges. The magnitude of these fields is described by a fundamental constant on the order of $10^{-41} \mathrm{~kg}^{-1}$. The same interactions can be observed in the solar system, where they lead to small changes in planetary orbits.
\end{abstract}

Keywords galactic rotation curves, dark matter, solar system, perihelion of Mercury, nodes of Venus

PACS numbers 95.30.Sf, 98.62.-g

Observations in radio astronomy of the $21 \mathrm{~cm}$ hydrogen line in interstellar gas and its broadening due to the relative motion of hydrogen clouds in interstellar space within galaxies have been employed since the 1960s to measure the rotational velocities of galaxies relative to a galactic center [1-3]. Based on the distribution of stellar mass in the galaxies, it was expected that the rotational velocity would roughly decrease with the inverse square root of the distance from the center [4]. However, observations by Rubin established that the velocity does not decrease, but rather remains constant with increasing distance or slightly increases [2, 4], as shown in Fig. 1. This fact poses a substantial challenge for our current understanding of nature at this scale, as it either implies that we do not understand what a large part of our universe is composed of (the mass unaccounted for is more than five times the baryonic mass in the universe $[5,6]$ ) or that we do not understand how masses interact (neither Newton's nor Einstein's formulation of the law of gravitation accounts for the discrepancy). This discrepancy was originally termed the "missing matter" problem, which has since morphed into the dark-matter problem [6], as it is thought that its solution might be obtained through a genuinely new form of matter in the universe that does not interact in conventional ways. The concept of dark matter, as Milgrom pointed out [7], is based on three separate assumptions: (i) the force governing the dynamics of interstellar hydrogen is gravity, (ii) the gravitational force depends on the source of the gravity field and the mass of the particle, and (iii) Newtons second law. Milgrom modified Newton's second law to read:

$$
m_{g} \mu\left(\frac{a}{a_{0}}\right) \boldsymbol{a}=\boldsymbol{F},
$$

where $m_{g}$ is the mass of hydrogen, $\mu$ is a function depending on acceleration $a$ and a constant $a_{0}$, and $\boldsymbol{F}$ is the force due to acceleration $\boldsymbol{a}$. For accelerations much larger than $a_{0}=2 \times 10^{-10} \mathrm{~m} \cdot \mathrm{s}^{-2}$, Newtonian dynamics is restored, while for smaller acceleration, the relation leads to a constant orbital velocity $V=\sqrt[4]{G M a_{0}}$, where $G$ is the gravitational constant and $M$ the mass of a galaxy [7]. However, Eq. (1) is only a "working formula, of limited validity $[\cdots]$ and does not constitute a theory" [8]. Moreover, if one considers the whole of physical theory and not just the essentially static laws of gravitation formulated here, then assumption (i) may not be strictly valid. The reason for such an assumption can be found in electrodynamics [9], where charges interact through two separate fields: they interact either through electrostatic Coulomb fields, equivalent to gravitational interactions of mass, or through magnetic fields, which currently have no equivalent in the theory of mass dynamics. It will be shown that such a field, which we call the rotor field $\boldsymbol{R}$, leads to identical results, suitable to account for the 


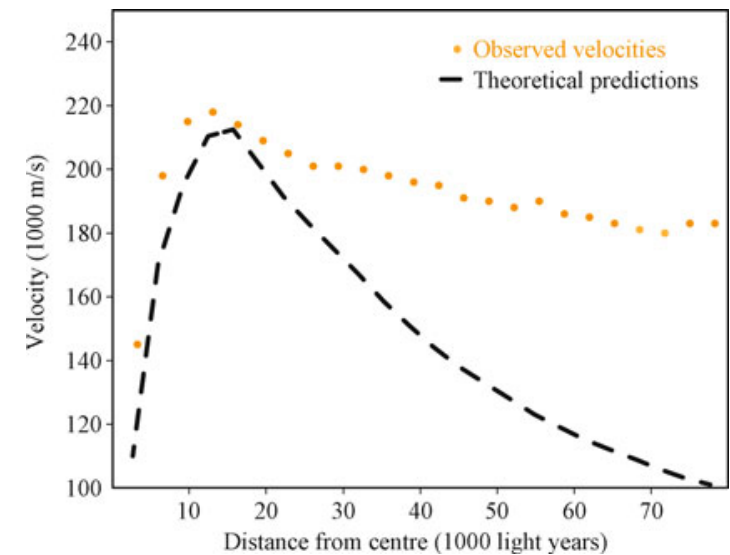

Fig. 1 Observations and theoretical predictions for the galaxy NGC 2903. Observed (orange dots) and predicted (broken black line) velocity of interstellar hydrogen in the galaxy. The predictions are based on gravity and the observed stellar mass (data from Ref. [4]).

dark-matter problem.

The concept of rotor fields and forces is similar to the modified Newtonian dynamics in that it provides a solution to the problem with a change in interactions of moving mass, rather than with additional mass components. Given that no credible candidates for these additional components have been found or can even be envisaged at present, an approach based on interactions is at once safer and more grounded in scientific facts. In contrast to the modified Newtonian dynamics, the concept of rotor fields and rotor forces leaves the general validity of Newtonian mechanics unquestioned, i.e., it does not attempt to assert that the basic physical laws are only valid for a limited range of accelerations. Moreover, by providing a general framework for the interactions of rotating mass via fields and forces due to rotations, it also allows for a generalization of the concept to other environments, such as the solar system. Therefore, it has the additional advantage of being more widely applicable.

Galaxies can have many different shapes. Here, we concentrate on symmetrical disk-shaped galaxies. The reason for this restriction is that excellent data of such galaxies exist in the work of Begeman et al. [4] and that simplifications are necessary to keep the new theoretical model as transparent and simple as possible. A galactic disk is a two-dimensional system, as shown in Fig. 2. The following model of stellar dynamics is based on two discrete assumptions: (i) mass in angular rotation around the galactic center generates a rotor field, which is radiated outward in the plane of the galaxy, and (ii) the field amplitude linearly decreases with increasing distance from the center. The second assumption is the application of Gauss's law to a two-dimensional system [9]. Because the circumference of a circle of radius $r$ is $2 \pi r$, the field amplitude $\boldsymbol{R}$ must be inversely proportional to $r$. Such systems are not unknown in electrodynamics, as the field of antennas is commonly very directional [9]. The field $\boldsymbol{R}$ for positive rotation can then be described by:

$$
\boldsymbol{R}(r)=\mu_{R} \frac{\boldsymbol{e}_{z}}{r} \int \mathrm{d} r^{\prime} \rho\left(r^{\prime}\right) 2 \pi r^{\prime} V\left(r^{\prime}\right) .
$$

Here, $\mu_{R}$ is the rotor constant, which will be determined from astronomical data, $\boldsymbol{e}_{z}$ is a unit vector in the $z$ direction, $\rho\left(r^{\prime}\right)$ is the two-dimensional mass density, and $V\left(r^{\prime}\right)$ is the angular velocity of the mass distribution. As the mass density of a galaxy is usually concentrated near the center and decreases with the square of the distance from the center [4], we may further simplify the equation by assuming that most of the galactic mass $M$ is contained within a certain radius $r_{0}$, and that the velocity distribution of this mass can be replaced by a constant $V_{0}$. This simplifies Eq. (2) to:

$$
\boldsymbol{R}=\mu_{R} \frac{\boldsymbol{e}_{z}}{r} M V_{0}
$$

In electrodynamics, the interaction between a magnetic field and charge in motion is described by the Lorentz force [9]. We assume that a similar law holds for the interaction between a rotor field and mass in motion. For a velocity vector in the $\varphi$-direction, owing to the circular rotation around the galactic center, $\boldsymbol{V}(r)=r \cdot \omega(r) \boldsymbol{e}_{\varphi}$, and the resulting rotor force $\boldsymbol{F}_{R}$ on a unit mass will be directed inward along the radial vector $\boldsymbol{e}_{r}$ and will be equal to the centrifugal forces directed outward, if $\omega(r)=V_{0} / r$. Hence,

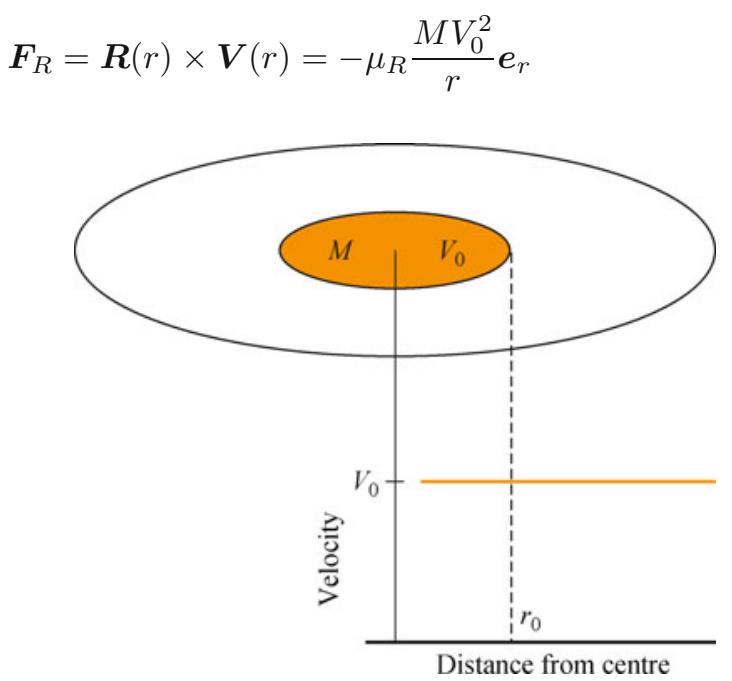

Fig. 2 Effect of rotor fields in a simple model. The spiral galaxy is simplified to a disk. The orange circle indicates the cutoff radius for the central rotating mass, and the velocity distribution due to rotor fields and their interaction will be flat in the outer regions of the disk. 
for a suitably chosen value of the rotor constant $\mu_{R}$. The result is the same as with the modified Newtonian dynamics: while gravity as described by Newton's or Einstein's theory of gravitation will lead to a decreasing angular velocity as the distance from the center increases, this model of rotor fields and dynamic interactions yields a flat velocity distribution. The rotor constant $\mu_{R}$ can be calculated from existing data with the relationship between velocities $V$, galactic mass $M$, and acceleration constant $a_{0}$. For many galaxies, the observed velocity is between 100 and $300 \mathrm{~km} / \mathrm{s}$. In our simple model, this implies that $\mu_{R}$ is between $10^{-40} \mathrm{~kg}^{-1}$ and $10^{-42} \mathrm{~kg}^{-1}$. Therefore, the forces related to these interactions are comparatively small. While the model explains why rotational velocity remains flat in the outer regions of a disk-shaped galaxy, it does not account for the TullyFisher relation between the mass of a galaxy and rotational velocity [10]. Here, it must be considered that mass rotating near the center of a galaxy generates a rotor field, which is radiated outward. Then, the question arises as to whether rotor fields will influence rotations near the center as well. There has to be some radius $r^{\prime}=r_{0} / x$, where the rotor fields of matter rotating inside will determine the dynamics of mass rotating outside, while inside this radius, dynamics will be determined mainly by gravitational attraction. The mass inside $r^{\prime}$ is $M / x^{2}$; therefore, the equivalence of centrifugal and gravitational forces leads to:

$$
V_{0}^{2}=\frac{G M}{r_{0} x}
$$

Empirically, it is known that $V_{0}$ increases with increasing $r_{0}$ of a galaxy. In the simplest case, the relation between $V_{0}$ and $r_{0}$ will be linear. It is also plausible that the radius $r^{\prime}=r_{0} / x$ will not vary too much for different galaxies; that is, it will not increase in the same way as $r_{0}$. To a first approximation, this implies that $x$ is proportional to $r_{0}$. Because $r_{0}$ is proportional to $V_{0}$, the product $x r_{0}$ is proportional to $V_{0}^{2}$. Setting the constant equal to $a_{0}^{-1}$, we recover the previous result. However, it is likely that $a_{0}$ will vary for different galaxies, and astronomical data show that the velocity does not exactly follow a fourthpower law [10]; i.e., this derivation of the Tully-Fisher relation is only an approximation.

If rotor fields and their interactions are real, then they must apply to all rotating masses, as well as to the mass within the solar system. In the solar system, the dominant mass is that of the sun. At $1.99 \times 10^{30} \mathrm{~kg}$, the solar mass [11] is approximately ten orders of magnitude smaller than the mass of a galaxy; effects within this system on planetary orbits will consequently be much smaller. The mass of the sun rotates with a period vary- ing between 25 days (equator) and 35 days (near the poles) [11]. In the following, we assume rigid rotation with a period of 26 days, and we assume that the density of solar mass is constant. For $\omega_{S}=4.45 \times 10^{-7} \mathrm{~s}^{-1}$ and a solar radius $r_{S}=6.96 \times 10^{8} \mathrm{~m}$, the rotor field in the plane perpendicular to the solar axis of rotation will be

$$
\boldsymbol{R}(r)=\mu_{R} \frac{\boldsymbol{e}_{z}}{r} 3.63 \times 10^{32} \mathrm{~kg} \cdot \mathrm{s}^{-1} .
$$

Consequently, the force on a unit mass at the position of the Mercurial or terrestrial orbit is $\mu_{R} \cdot 4.78 \times 10^{25} \mathrm{~N} / \mathrm{kg}$ or $\mu_{R} \cdot 1.15 \times 10^{25} \mathrm{~N} / \mathrm{kg}$, respectively. To determine the effect on a planetary orbit, we assume that the additional force will manifest itself as an additional centripetal force, which will lead to a slight increase in the orbital velocity of mass. For $\mu_{R}=10^{-40} \mathrm{~kg}^{-1}$, we obtain an additional velocity of $16.6 \mathrm{~mm} / \mathrm{s}$ (Mercury) and $13.1 \mathrm{~mm} / \mathrm{s}$ (Earth) [12]. From the given velocities, we calculate the orbital shifts in one terrestrial year as 1.87 and 0.57 arc seconds, respectively. The value for the rotation of the mercurial orbit of 187 arc seconds per century is approximately four times the value calculated with general relativity [13]. The assumption that the perihelion advance of Mercury, as shown in Fig. 3, is due to rotor fields would allow us to calibrate the rotor field constant. For 43 arc seconds per century, the value of $\mu_{R}=0.53 \times 10^{-41} \mathrm{~kg}^{-1}$. This value is well within the range determined from the dynamics of galaxies. The advance of the terrestrial orbit will then be 13 arc seconds or approximately three times the effect calculated with general relativity.

An identical calculation can be made for the Venutian orbit [14], which yields a result of 12 arc seconds for the orbit rotation. It has indeed been observed that the nodes of Venus move by approximately 10 arc seconds per century [15], an observation which cannot be

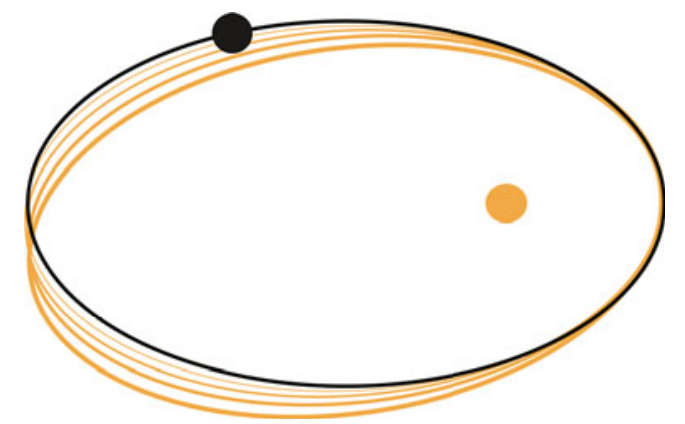

Fig. 3 Effect of solar rotation on the planets of the solar system. Black: Stable elliptical orbit resulting from the gravitational pull of a central mass. Orange: Mercury's elliptical orbit will rotate around the sun, and the rotation is visible as a gradual advance of the perihelion, which is the point of closest approach to the sun. Note that most of the advance is due to the gravitational pull of solar planets; only a small fraction of 43 arc seconds remains unexplained by Newton's gravitational model. 
explained by general relativity and is indeed accounted for - within the errors likely to arise from the simplification of this model - in the model of rotor fields and rotor forces. Because the effect of rotor fields will lead to flat velocity distributions with increasing distance from the center of the solar system, as explained above, we expect similar deviations from current theories on gravitation as well for the outer regions of the system. In summary, the model of rotor fields and rotor forces can account for three separate effects:

1) The flat velocity distributions of interstellar hydrogen in galaxies.

2) The advance of the perihelion of Mercury.

3) The advance of the nodes of Venus.

General relativity, by contrast, can only account for one of the three effects (Mercury's perihelion) and, most importantly, completely fails to explain the rotational velocities within galaxies.

It is surprising that by using the missing-matter problem as a starting point, one can account for the problem by postulating a new form of mass interactions, which has observable consequences in our immediate vicinity. The predicted changes of planetary orbits also agree reasonably well with astronomical data and with theoretical predictions made in a completely different context. At this point, a legitimate question arises as to whether the model of rotor fields and forces aims at providing an alternative explanation for the advance of planetary orbits to the one currently accepted by the scientific community, i.e., the explanation forwarded by the theory of general relativity. Strictly speaking, this question cannot be answered at present. We only show with this model that the question of the observed rotational velocities in distant galaxies may not be unrelated to the question of how planetary orbits are affected by the rotation of the sun. The question of whether the advance of Mercury's orbit is caused by the rotation of solar mass or the distortion of the flat space-time geometry due to solar mass will have to be addressed in the future. However, it is quite astonishing that the fundamental constant governing one effect - rotational velocities in galaxies - is within a certain range, making it plausible that it may also govern the other effect: the rotation of planetary orbits.

If it is established that a relation between these two effects exists, then it seems possible that this model will open up astrophysics to a truly dynamical analysis of mass interactions in the same manner classical electrodynamics was made possible by the discovery of magnetic fields and their interactions. However, it cannot be ex- pected at this point that the application of this model will be able to account for every astronomical observation of stellar dynamics without additional development. Moreover, as shown by the estimate of the effect of solar rotation, rotor fields arise from the orbital velocity of mass around the galactic center and the rotation of mass in individual stellar objects. In practice, this will lead to a large variability in the dynamics of individual galaxies.

In summary, we have shown that a new mode of interactions of mass in motion leads to the prediction of flat velocity distributions in galaxies and a dependency of this velocity on galactic mass. The results agree with astronomical observations and account for the problem known as the dark-matter problem. Similar interactions play a role in the solar system, where they lead to predictions for the advance of planetary orbits in agreement with astronomical observations.

Acknowledgements The author acknowledges EPSRC support for the UKCP consortium, grant No. EP/K013610/1.

Open Access This article is distributed under the terms of the Creative Commons Attribution License which permits any use, distribution, and reproduction in any medium, provided the original author(s) and the source are credited.

\section{References and notes}

1. H. I. Ewen and E. M. Purcell, Observation of a line in the galactic radio spectrum: radiation from galactic hydrogen at 1420 Mc./sec., Nature 168, 356 (1951)

2. Vera C. Rubin and W. Kent Ford Jr., Rotation of the Andromeda Nebula from a spectroscopic survey of emission regions, Astrophys. J. 159, 379 (1970)

3. H. J. Rood, Clusters of galaxies, Rep. Prog. Phys. 44(10), 1077 (1981)

4. K. G. Begeman, A. H. Broeils, and R. H. Sanders, Extended rotation curves of spiral galaxies - Dark haloes and modified dynamics, Mon. Not. R. Astron. Soc. 249, 523 (1991)

5. See, for example, the dark matter focus on the NASA website: http://science.nasa.gov/astrophysics/focusareas/what-is-dark-energy/

6. X.-J. Bi, P.-F. Yin, and Q. Yuan, Status of dark matter detection, Front. Phys. 8(6), 794 (2013)

7. M. Milgrom, A modification of the Newtonian dynamics as a possible alternative to the hidden mass hypothesis, Astrophys. J. 270, 365 (1983)

8. M. Milgrom, A modification of the Newtonian dynamics Implications for galaxies, Astrophys. J. 270, 371 (1983)

9. J. D. Jackson, Classical Electrodynamics, 3rd Ed., NJ: Wiley, 1998

10. S. Torres-Flores, B. Epinat, P. Amram, H. Plana, C. Mendes de Oliveira, GHASP: An $\mathrm{H} \alpha$ kinematic survey of spiral and 
irregular galaxies - IX: The near-infrared, stellar and baryonic Tully-Fisher relations, Mon. Not. R. Astron. Soc. 416, 1936 (2011)

11. See the NASA website at: http://nssdc.gsfc.nasa.gov/planetary/factsheet/

12. For the calculations we assumed circular orbits, with $r_{M}=$ $5.79 \times 10^{10} \mathrm{~m}$ and $\omega_{M}=1.32 \times 10^{-7} \mathrm{~s}^{-1}$ for Mercury, and

$$
r_{E}=1.50 \times 10^{11} \mathrm{~m} \text { and } \omega_{E}=3.17 \times 10^{-8} \mathrm{~s}^{-1} \text { for Earth. }
$$

13. G. M. Clemence, The relativity effect in planetary motions, Rev. Mod. Phys. 19, 361 (1947)

14. For the calculations of Venus we assumed a circular orbit with $r_{V}=1.08 \times 10^{11} \mathrm{~m}$ and $\omega_{V}=5.15 \times 10^{-8} \mathrm{~s}^{-1}$.

15. Jean Chazy, La Theorie de la relativite et la Mechanique celeste, Gauthier Villars, Paris, 1928, p. 230 